\title{
Convex Drawings of Plane Graphs of Minimum Outer Apices
}

\author{
Kazuyuki Miura ${ }^{1}$, Machiko Azuma ${ }^{2}$, and Takao Nishizeki ${ }^{2}$ \\ 1 Faculty of Symbiotic Systems Science, \\ Fukushima University, Fukushima 960-1296, Japan \\ miura@sss.fukushima-u.ac.jp \\ 2 Graduate School of Information Sciences, \\ Tohoku University, Sendai 980-8579, Japan \\ azuma@nishizeki.ecei.tohoku.ac.jp, nishi@ecei.tohoku.ac.jp
}

\begin{abstract}
In a convex drawing of a plane graph $G$, every facial cycle of $G$ is drawn as a convex polygon. A polygon for the outer facial cycle is called an outer convex polygon. A necessary and sufficient condition for a plane graph $G$ to have a convex drawing is known. However, it has not been known how many apices of an outer convex polygon are necessary for $G$ to have a convex drawing. In this paper, we show that the minimum number of apices of an outer convex polygon necessary for $G$ to have a convex drawing is, in effect, equal to the number of leaves in a triconnected component decomposition tree of a new graph constructed from $G$, and that a convex drawing of $G$ having the minimum number of apices can be found in linear time.
\end{abstract}

\section{Introduction}

Recently automatic aesthetic drawing of graphs has created intense interest due to their broad applications, and as a consequence, a number of drawing methods have come out $1,2,3,4,8$. The most typical drawing of a plane graph $G$ is a straight line drawing in which all vertices of $G$ are drawn as points and all edges are drawn as straight line segments without any edge-intersection. A straight line drawing of $G$ is called a convex drawing if every facial cycle is drawn as a convex polygon, as illustrated in Fig. 1, 1, 9.

In a convex drawing of a plane graph $G$, the outer facial cycle $F_{\mathrm{o}}(G)$ of $G$ must be drawn as a convex polygon. A polygonal drawing $F_{\mathrm{o}}^{*}$ of $F_{\mathrm{o}}(G)$, called an outer convex polygon, plays a crucial role in finding a convex drawing of $G$. The plane graph $G$ in Fig. 2(a) admits a convex drawing if an outer convex polygon $F_{\mathrm{o}}^{*}$ has four or more apices as illustrated in Fig. 2(b), where apices are drawn as white circles. However, if $F_{\mathrm{o}}^{*}$ has only three apices, that is, $F_{\mathrm{o}}^{*}$ is a triangle, then $G$ does not admit a convex drawing as illustrated in Fig. 2(c).

A necessary and sufficient condition for a plane graph $G$ to have a convex drawing is known 1, 9. A linear-time algorithm is also known for finding a convex drawing of $G$ if $G$ satisfies the condition [1,7]. We recently give a necessary and sufficient condition for a plane graph $G$ to have a convex drawing such that 


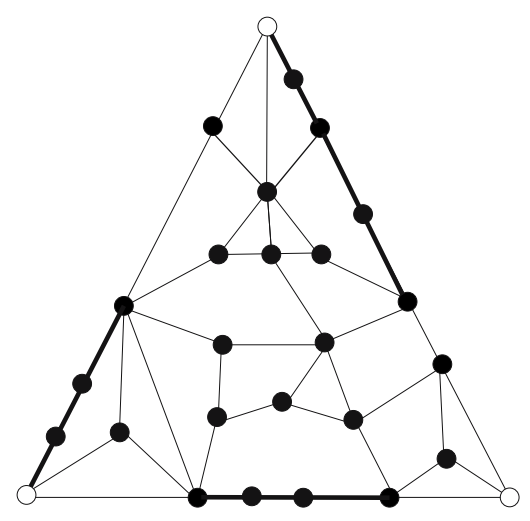

Fig. 1. Convex drawing of a plane graph

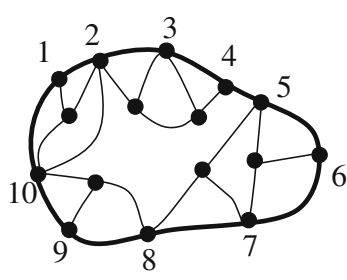

(a)

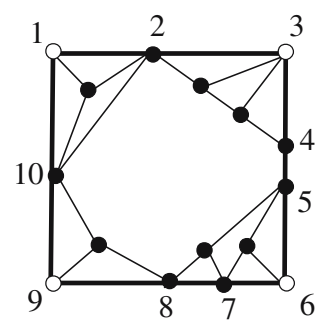

(b)

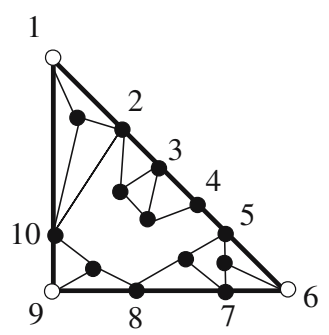

(c)

Fig. 2. (a) Plane graph, (b) convex drawing, and (c) non-convex drawings

an outer convex polygon $F_{0}^{*}$ has exactly three apices 6]. However, it is not known how many apices of $F_{\mathrm{o}}^{*}$ are necessary for a plane graph $G$ to have a convex drawing.

In this paper, we show that the minimum number of apices of an outer convex polygon $F_{\mathrm{o}}^{*}$ necessary for $G$ to have a convex drawing is, in effect, equal to the number of leaves in a triconnected component decomposition tree of a new graph constructed from $G$, and that a convex drawing of $G$ having the minimum number of apices of $F_{\mathrm{o}}^{*}$ can be found in linear time.

The remainder of the paper is organized as follows. In Section 2 we give some definitions and two known lemmas. In Section 3 we present our results. Finally we conclude in Section 4.

\section{Preliminaries}

In this section, we give some definitions and two known lemmas.

We denote by $G=(V, E)$ an undirected connected simple graph with vertex set $V$ and edge set $E$. An edge joining vertices $u$ and $v$ is denoted by $(u, v)$. The 
degree of a vertex $v$ in $G$ is the number of neighbors of $v$ in $G$, and is denoted by $d(v)$.

A graph is planar if it can be embedded in the plane so that no two edges intersect geometrically except at a vertex to which they are both incident. A plane graph is a planar graph with a fixed embedding. A plane graph $G$ divides the plane into connected regions, called faces. We denote by $F_{\mathrm{o}}(G)$ the outer face of $G$. The boundary of $F_{\mathrm{o}}(G)$ is also denoted by $F_{\mathrm{o}}(G)$. A vertex on $F_{\mathrm{o}}(G)$ is called an outer vertex, while a vertex not on $F_{\mathrm{o}}(G)$ is called an inner vertex. An edge on $F_{\mathrm{o}}(G)$ is called an outer edge, while an edge not on $F_{\mathrm{o}}(G)$ is called an inner edge.

A polygonal drawing $F_{\mathrm{o}}^{*}$ of $F_{\mathrm{o}}(G)$ is called an outer convex polygon if $F_{\mathrm{o}}^{*}$ is a convex polygon. A (geometric) vertex of a polygon $F_{\mathrm{o}}^{*}$ is called an outer apex. An outer convex polygon $F_{\mathrm{o}}^{*}$ is extendable if $G$ has a convex drawing in which $F_{\mathrm{o}}(G)$ is drawn as the convex polygon $F_{\mathrm{o}}^{*}$. For example, the outer rectangle drawn by thick lines in Fig. 2(b) is extendable, while the outer triangle in Fig. 2(c) is not extendable. We denote by $G-F_{\mathrm{o}}(G)$ the graph obtained from $G$ by deleting all outer vertices.

We call a vertex $v$ of a connected graph $G$ a cut vertex if its removal from $G$ results in a disconnected graph. A connected graph $G$ is biconnected if $G$ has no cut vertex. If $G$ has a convex drawing, then $G$ is biconnected.

The following necessary and sufficient condition for a plane graph $G$ to have a convex drawing is known.

Lemma 1. [1,9] Let $G$ be a biconnected plane graph, and let $F_{\mathrm{o}}^{*}$ be an outer convex polygon of $G$. Assume that $F_{\mathrm{o}}^{*}$ is a $k$-gon, $k \geq 3$, and that $P_{1}, P_{2}, \cdots, P_{k}$ are the $k$ paths in $F_{\mathrm{o}}(G)$, each corresponding to a side of the polygon $F_{\mathrm{o}}^{*}$. Then $F_{\mathrm{o}}^{*}$ is extendable if and only if the following Conditions (a)-(c) hold.

(a) For each inner vertex $v$ with $d(v) \geq 3$, there exist three paths disjoint except $v$, each joining $v$ and an outer vertex;

(b) The graph $G-F_{\mathrm{o}}(G)$ has no connected component $H$ such that all the outer vertices adjacent to vertices in $H$ lie on a single path $P_{i}$, and no two outer vertices in each path $P_{i}$ are joined by an inner edge (see Fig. 3); and

(c) Every cycle containing no outer edge has at least three vertices of degree $\geq 3$.

We call a pair $\{u, v\}$ of vertices in a biconnected graph $G$ a separation pair if its removal from $G$ results in a disconnected graph, that is, $G-\{u, v\}$ is not connected. A biconnected graph $G$ is triconnected if $G$ has no separation pair. A plane biconnected graph $G$ is internally triconnected if, for any separation pair $\{u, v\}$ of $G$, both $u$ and $v$ are outer vertices and each connected component of $G-\{u, v\}$ contains an outer vertex. In other words, $G$ is internally triconnected if and only if it can be extended to a triconnected graph by adding a vertex in an outer face and joining it to all outer vertices. If a biconnected plane graph $G$ is not internally triconnected, then $G$ has a separation pair $\{u, v\}$ illustrated in Figs. 4(a)-(c) and a "split graph" $H$ contains an inner vertex other than $u$ and $v$. 


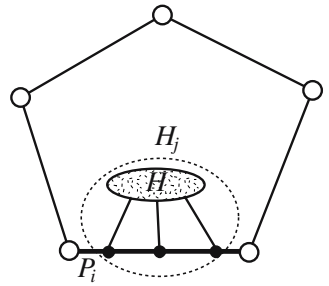

(a)

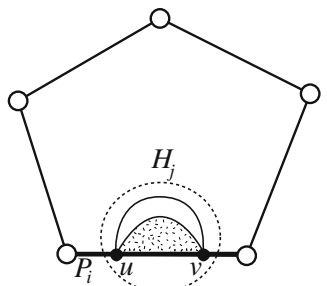

(b)

Fig. 3. Examples violating Condition (b)

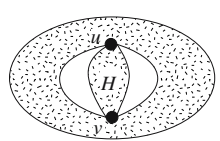

(a)

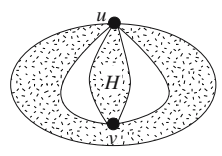

(b)

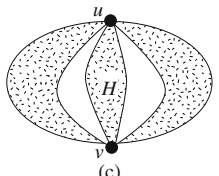

(c)

Fig. 4. Biconnected plane graphs which are not internally triconnected

Let $G=(V, E)$ be a biconnected graph, and let $\{u, v\}$ be a separation pair of $G$. Then, $G$ has two subgraphs $G_{1}^{\prime}=\left(V_{1}, E_{1}^{\prime}\right)$ and $G_{2}^{\prime}=\left(V_{2}, E_{2}^{\prime}\right)$ such that

(a) $V=V_{1} \cup V_{2}, V_{1} \cap V_{2}=\{u, v\}$; and

(b) $E=E_{1}^{\prime} \bigcup E_{2}^{\prime}, E_{1}^{\prime} \bigcap E_{2}^{\prime}=\emptyset,\left|E_{1}^{\prime}\right| \geq 2,\left|E_{2}^{\prime}\right| \geq 2$.

The graph $G$ in Fig. [5) (a) has six separation pairs $\left\{u_{1}, u_{2}\right\},\left\{u_{1}, u_{3}\right\},\left\{u_{2}, u_{3}\right\}$, $\left\{u_{2}, u_{7}\right\},\left\{u_{3}, u_{6}\right\}$, and $\left\{u_{4}, u_{5}\right\}$.

For a separation pair $\{u, v\}$ of $G, G_{1}=\left(V_{1}, E_{1}^{\prime}+(u, v)\right)$ and $G_{2}=\left(V_{2}, E_{2}^{\prime}+\right.$ $(u, v))$ are called the split graphs of $G$ with respect to $\{u, v\}$. The new edges $(u, v)$ added to $G_{1}$ and $G_{2}$ are called the virtual edges. Even if $G$ has no multiple edges, $G_{1}$ and $G_{2}$ may have. Dividing a graph $G$ into two split graphs $G_{1}$ and $G_{2}$ are called splitting. Reassembling the two split graphs $G_{1}$ and $G_{2}$ into $G$ is called merging. Merging is the inverse of splitting. Suppose that a graph $G$ is split, the split graphs are split, and so on, until no more splits are possible, as illustrated in Fig. 5.(b) where virtual edges are drawn by dotted lines. The graphs constructed in this way are called the split components of $G$. The split components are of three types: triple bonds (i.e. a set of three multiple edges), triangles, and triconnected graphs. The triconnected components of $G$ are obtained from the split components of $G$ by merging triple bonds into a bond and triangles into a ring, as far as possible, where a bond is a set of multiple edges and a ring is a cycle. The graph in Fig. 5(a) is decomposed into seven triconnected components $H_{1}, H_{2}, \cdots, H_{7}$ as depicted in Fig. 5)(c), where $H_{1}, H_{2}$ and $H_{6}$ are triconnected graphs, $H_{3}, H_{4}$ and $H_{7}$ are rings, and $H_{5}$ is a bond. The split components of $G$ are not necessarily unique, but the triconnected components of $G$ are unique [5].

Let $T$ be a tree in which each node corresponds to a triconnected component $H_{i}$ and $T$ has an edge $\left(H_{i}, H_{j}\right), i \neq j$, if and only if $H_{i}$ and $H_{j}$ are triconnected components with respect to the same separation pair, as illustrated in Fig. 5 (d). 


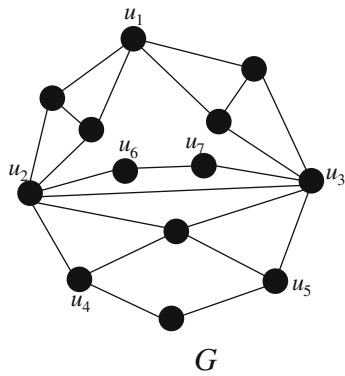

(a)
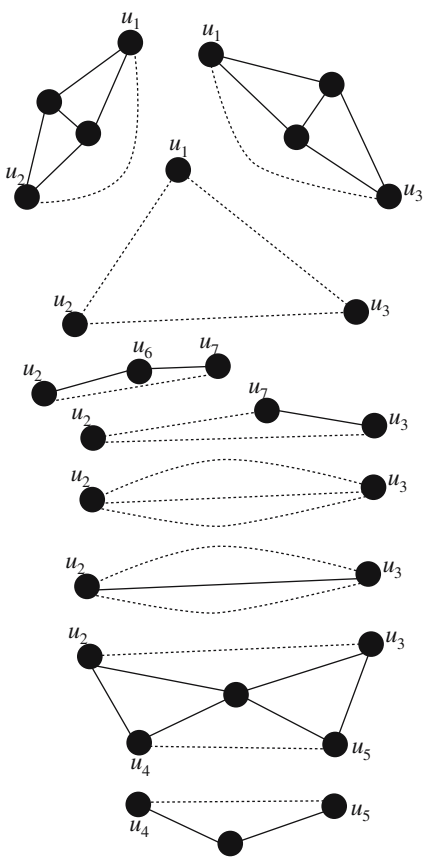

(b)
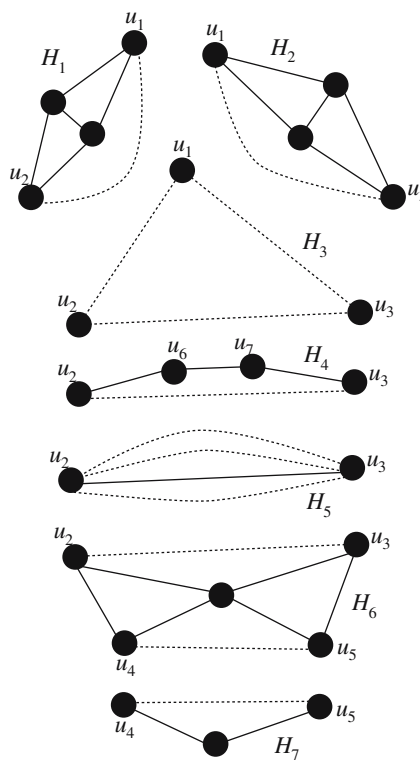

(c)

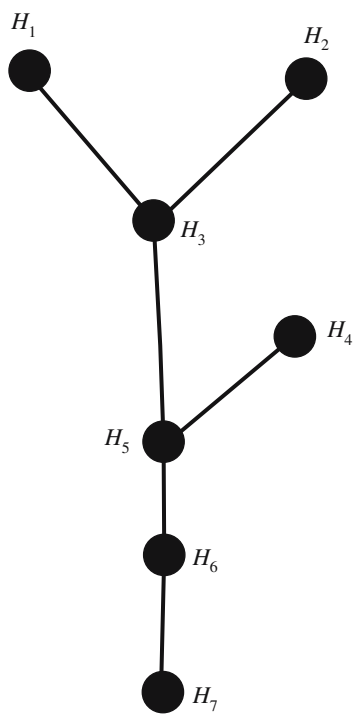

(d)

Fig. 5. (a) A biconnected graph $G$, (b) split components, (c) triconnected components, and (d) a triconnected component decomposition tree $T$ of $G$ 
We call $T$ a triconnected component decomposition tree of $G$ [5]. Clearly every leaf of $T$ does not correspond to a bond, but corresponds to a triconnected graph or a ring.

The following lemma is known.

Lemma 2. 5] A triconnected component decomposition tree $T$ of a graph $G$ can be found in linear time.

\section{Convex Drawing of Minimum Outer Apices}

One may obviously assume that a plane graph $G$ is biconnected. One may further assume that every inner vertex of $G$ has degree three or more; if an inner vertex $v$ has degree two in $G$, then delete $v$ from $G$, join the neighbors of $v$, and let $G^{\prime}$ be the resulting graph; clearly $G$ has a convex drawing if and only if $G^{\prime}$ has no multiple edges and has a convex drawing. We can then newly formalize a necessary and sufficient condition for $G$ to have a convex drawing, as follows.

Theorem 1. Let $G$ be a plane biconnected graph in which every inner vertex has degree three or more, and let $T$ be a triconnected component decomposition tree of $G$. Then the following (a)-(c) are equivalent with each other:

(a) $G$ has a convex drawing;

(b) $G$ is internally triconnected; and

(c) both of every separation pair are outer vertices, and every node of $T$ corresponding to a bond has degree two in $T$.

Proof. We verify $(\mathrm{a}) \Leftrightarrow(\mathrm{b})$ and $(\mathrm{b}) \Leftrightarrow(\mathrm{c})$, as follows.

$(\mathrm{a}) \Rightarrow(\mathrm{b})$ : Assume that $G$ has a convex drawing. Then $G$ has an extendable outer convex polygon $F_{\mathrm{o}}^{*}$, and hence Conditions (a)-(c) in Lemma 1 hold for $F_{\mathrm{o}}^{*}$. Suppose for a contradiction that $G$ is not internally triconnected. Then $G$ has a separation pair $\{u, v\}$ illustrated in Figs. 4(a)-(c). In a split graph $H$ with respect to $\{u, v\}$, there is an inner vertex $x$ other than $u$ and $v$. Vertex $x$ has degree three or more, and hence every path joining $x$ and an outer vertex must pass through either $u$ or $v$. Therefore there are no three paths disjoint except $x$, each joining $x$ and an outer vertex. Thus Condition (a) does not hold, a contradiction.

$(\mathrm{b}) \Rightarrow(\mathrm{a})$ : Assume that $G$ is internally triconnected. Let $F_{\mathrm{o}}^{*}$ be an outer convex polygon in which every outer vertex of $G$ is an apex of $F_{\mathrm{o}}^{*}$. We show that $F_{\mathrm{o}}^{*}$ is extendable and hence $G$ has a convex drawing. Since $G$ is internally triconnected, clearly both Conditions (a) and (c) in Lemma 1 hold. Thus we shall show that Condition (b) holds for $F_{\mathrm{o}}^{*}$. Each path $P_{i}$ in $F_{\mathrm{o}}$, corresponding to a side of $F_{\mathrm{o}}^{*}$, consists of a single edge, and $G$ is a simple graph. Therefore the two outer vertices in $P_{i}$ are not joined by an inner edge. Since $G$ is internally triconnected, one can easily know that $G-F_{\mathrm{o}}(G)$ has no connected component $H$ such that all the outer vertices adjacent to vertices in $H$ lie on a single path $P_{i}$. Thus $G$ satisfies Conditions (a)-(c), and hence by Lemma $1 F_{\mathrm{o}}^{*}$ is extendable. 


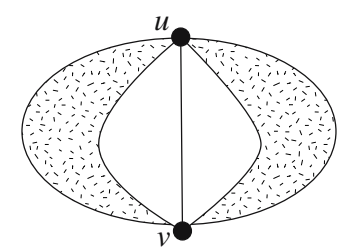

(a)

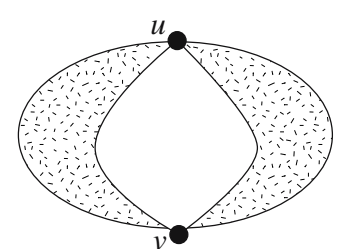

(b)

Fig. 6. Plane graphs having separation pairs corresponding to bonds

(b) $\Rightarrow(\mathrm{c})$ : Assume that $G$ is internally triconnected. Then both of every separation pair of $G$ are outer vertices, and $G$ does not have a separation pair $\{u, v\}$ illustrated in Figs. 4(a)-(c). Therefore, for every separation pair $\{u, v\}$, $G-\{u, v\}$ has exactly two connected components, each containing an outer vertex, as illustrated in Figs. 6(a) and (b); $G$ has an edge $(u, v)$ in Fig. 6) (a), while $G$ has no edge $(u, v)$ in Fig. 6(b). Hence one can easily observe that every node of $T$ corresponding to a bond has degree two in $T$; the bond for Fig. 6 (a) is a set of three multiple edges, two of which are virtual ones, and one of which is a real one; the bond for Fig. 6 (b) is a pair of virtual multiple edges.

$(c) \Rightarrow(b)$ : Assume that both of every separation pair are outer vertices, and every node of $T$ corresponding to a bond has degree two in $T$. We shall show that $G$ does not have a separation pair $\{u, v\}$ illustrated in Figs. 4(a)-(c). Since both of every separation pair are outer vertices, $G$ does not have a separation pair $\{u, v\}$ illustrated in Figs. 4(a) and (b). Assume for a contradiction that $G$ has a separation pair $\{u, v\}$ illustrated in Fig. 4(c). Then a node of $T$ corresponding to a bond containing virtual edges $(u, v)$ has degree three or more, a contradiction.

By Theorem 1 we may assume that $G$ is internally triconnected and each inner vertex of $G$ has degree three or more, as the graph in Fig. 1. Thus every vertex of degree two must be an outer vertex. Let $P=v_{0}, v_{1}, v_{2}, \cdots, v_{l+1}, l \geq 1$, be a path on $F_{\mathrm{o}}(G)$ such that $d\left(v_{0}\right) \geq 3, d\left(v_{1}\right)=d\left(v_{2}\right)=\cdots=d\left(v_{l}\right)=2$ and $d\left(v_{l+1}\right) \geq 3$. Such a path $P$ is called an outer chain of $G$. (The graph in Fig. 1 has four outer chains drawn by thick lines.) We then have the following lemma.

Lemma 3. Let $G$ be an internally triconnected plane graph, and let $P=$ $v_{0}, v_{1}, \cdots, v_{l+1}$ be an outer chain of $G$. Then the following Propositions (a) and (b) hold:

(a) If $G$ has an edge $\left(v_{0}, v_{l+1}\right)$, then $G$ has a convex drawing in which exactly one of the vertices $v_{1}, v_{2}, \cdots, v_{l}$ is an outer apex and $G$ has no convex drawing in which none of them is an outer apex.

(b) If $G$ has no edge $\left(v_{0}, v_{l+1}\right)$, then $G$ has a convex drawing in which none of the vertices $v_{1}, v_{2}, \cdots, v_{l}$ is an outer apex. (See Fig. 7.)

Proof. (a) Suppose that $G$ has an edge $\left(v_{0}, v_{l+1}\right)$. Since $G$ is internally triconnected, by Theorem $1 G$ has a convex drawing. Let $F_{\mathrm{o}}^{*}$ be the outer convex 


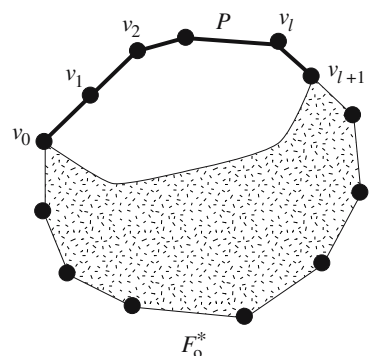

(a)

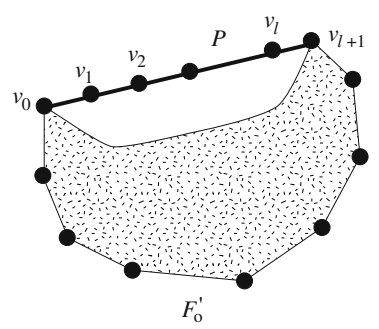

(b)

Fig. 7. Outer convex polygons $F_{\mathrm{o}}^{*}$ and $F_{\mathrm{o}}^{\prime}$

polygon of an arbitrary convex drawing of $G$. Clearly one or more of the vertices $v_{1}, v_{2}, \cdots, v_{l}$ are apices of $F_{\mathrm{o}}^{*}$. One can easily modify the drawing to a convex drawing in which exactly one of them is an outer apex.

(b) Suppose that $G$ has no edge $\left(v_{0}, v_{l+1}\right)$. Let $F_{\mathrm{o}}^{*}$ be the outer convex polygon of an arbitrary convex drawing of $G$. We may assume that at least one of the vertices $v_{1}, v_{2}, \cdots, v_{l}$ is an apex of $F_{\mathrm{o}}^{*}$, as illustrated in Fig. [7(a); otherwise, we have completed a proof for (b). We cut off the region enclosed by the path $P=v_{0}, v_{1}, \cdots, v_{l+1}$ and a straight line segment $v_{l+1}, v_{0}$ from the polygon $F_{\mathrm{o}}^{*}$. Let $F_{\mathrm{o}}^{\prime}$ be the resulting outer convex polygon, in which $v_{0}, v_{1}, \cdots, v_{l}$ lie on the line segment connecting $v_{0}$ and $v_{l+1}$, as illustrated in Fig. 7(b). We claim that $F_{\mathrm{o}}^{\prime}$ is extendable and hence $G$ has a convex drawing in which none of the vertices $v_{1}, v_{2}, \cdots, v_{l}$ is an apex of the outer convex polygon. Since $G$ is internally triconnected, both Conditions (a) and (c) in Lemma 1 hold for $F_{\mathrm{o}}^{\prime}$. Thus we shall show that Condition (b) holds for $F_{\mathrm{o}}^{\prime}$. Since Condition (b) holds for $F_{\mathrm{o}}^{*}$, the definition of $F_{\mathrm{o}}^{\prime}$ implies that Condition (b) holds for all paths of $F_{\mathrm{o}}^{\prime}$, other than $P$, each corresponding to a side of the polygon $F_{\mathrm{o}}^{\prime}$. Since vertices $v_{1}, v_{2}, \cdots, v_{l}$ have degree two and there is no edge $\left(v_{0}, v_{l+1}\right)$ in $G$, no two outer vertices in path $P$ are joined by an inner edge. Since $G$ is internally triconnected, $G-F_{\mathrm{o}}(G)$ has no connected component $H$ such that all the outer vertices adjacent to vertices in $H$ lie on the single path $P$. Thus $G$ satisfies Conditions (a)-(c), and hence $F_{\mathrm{o}}^{\prime}$ is extendable.

By Lemma 3, in order to find a convex drawing of minimum outer apices, we may "contract" an outer chain $P=v_{0}, v_{1}, \cdots, v_{l+1}$ of $G$ as follows: if $G$ has an edge $\left(v_{0}, v_{l+1}\right)$, then we replace $P$ in $G$ with an outer chain $P^{\prime}=v_{0}, v_{i}, v_{l+1}$ for an arbitrary index $i, 1 \leq i \leq l$; otherwise, we replace $P$ in $G$ with a single edge $\left(v_{0}, v_{l+1}\right)$. Contract every outer chain of $G$ as above, and let $G^{\prime}$ be the resulting graph. $G^{\prime}$ is called a contracted graph of $G$. Figure 8(b) illustrates a contracted graph of the graph $G$ in Fig. 8 (a). Since $G$ is internally triconnected, $G^{\prime}$ is also internally triconnected and hence $G^{\prime}$ has a convex drawing. One can easily observe that the following lemma holds.

Lemma 4. Let $G$ be an internally triconnected plane graph, and let $G^{\prime}$ be a contracted graph of $G$. If an outer convex polygon $F_{\mathrm{o}}^{*}$ is extendable for $G^{\prime}$ (as 


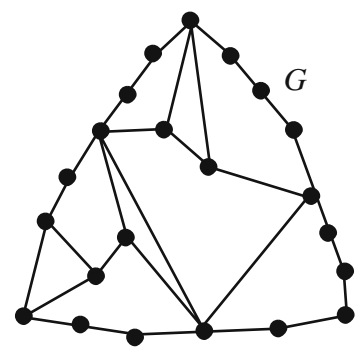

(a)

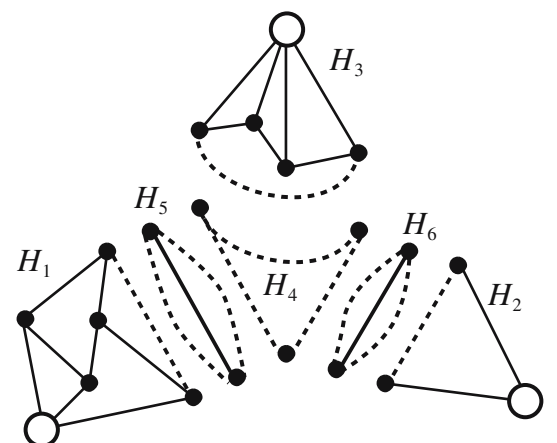

(c)

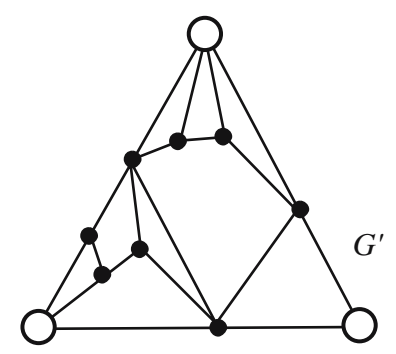

(e)

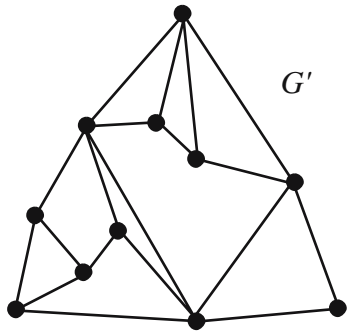

(b)

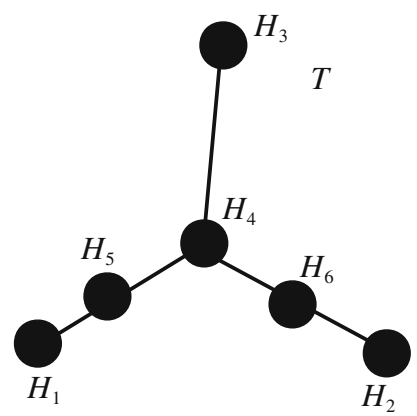

(d)

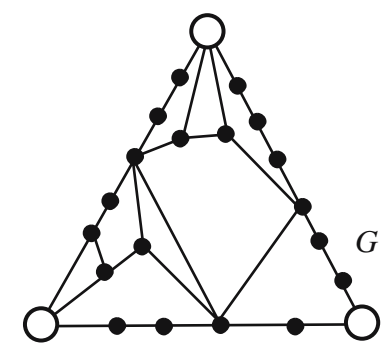

(f)

Fig. 8. (a) An internally triconnected graph $G$, (b) a contracted graph $G^{\prime}$ of $G$, (c) triconnected components of $G^{\prime}$, (d) a triconnected component tree $T$, (e) a convex drawing of $G^{\prime}$, and (f) a convex drawing of $G$

illustrated in Fig. 8(e)), then the same outer convex polygon $F_{\mathrm{o}}^{*}$ is extendable for $G$ (as illustrated in Fig. 8 (f)).

We now have the following lemma.

Lemma 5. Let $G$ be an internally triconnected plane graph, let $G^{\prime}$ be a contracted graph of $G$, let $T$ be a triconnected component decomposition tree of $G^{\prime}$, and let $n_{l}$ be the number of leaves of $T$. Then the minimum number of apices of an extendable convex polygon of $G^{\prime}$ is equal to $\max \left\{3, n_{l}\right\}$. 
Proof. If $n_{l}=1$, that is, $G^{\prime}$ is either a cycle or is a triconnected graph, then $G^{\prime}$ has a convex drawing whose outer facial cycle is drawn as a triangle [6, 8, Therefore, the minimum number of apices of an extendable convex polygon of $G^{\prime}$ is $\max \{3,1\}=3$. We may thus assume that $n_{l} \geq 2$.

We first claim that $G^{\prime}$ has no extendable outer convex polygon such that the number of apices is less than $\max \left\{3, n_{l}\right\}$. If $2 \leq n_{l} \leq 3$, then $\max \left\{3, n_{l}\right\}=3$ and hence $G^{\prime}$ has no extendable outer convex polygon such that the number of outer apices is less than $\max \left\{3, n_{l}\right\}$. We may thus assume that $n_{l} \geq 4$. Suppose for a contradiction that $G^{\prime}$ has an extendable outer convex polygon $F_{\mathrm{o}}^{*}$ having less than $\max \left\{3, n_{l}\right\}=n_{l}$ apices. Then $G^{\prime}$ has a triconnected component $H_{j}$, corresponding to a leaf of $T$, such that none of the outer vertices in $H_{j}$ other than the separation pair is an apex of $F_{\mathrm{o}}^{*}$. Hence all the outer vertices in $H_{j}$ lie on a single side $P_{i}$ of the polygon $F_{\mathrm{o}}^{*}$. Since $H_{j}$ corresponds to a leaf of $T, H_{j}$ is either a triconnected graph or a ring. $\left(H_{1}\right.$ and $H_{3}$ in Fig. 8(c) are triconnected graphs, while $H_{2}$ is a ring.) Consider first the case where $H_{j}$ is a triconnected graph. Then $G^{\prime}-F_{\mathrm{o}}\left(G^{\prime}\right)$ has a connected component such that all the outer vertices adjacent to vertices in the component lie on the side $P_{i}$, as illustrated in Fig. 3(a). Consider next the case where $H_{j}$ is a ring. Let $H_{p}$ be the triconnected component corresponding to the node of $T$ which is adjacent in $T$ to the leaf corresponding to $H_{j}$. By the definitions of $T$ and $G^{\prime}, H_{p}$ must be a bond and contain an inner edge joining two outer vertices of $G^{\prime}$. Therefore the two outer vertices on the side $P_{i}$ are joined by the inner edge. Thus, in either case, Condition (b) does not hold for $F_{\mathrm{o}}^{*}$. Hence $F_{\mathrm{o}}^{*}$ is not extendable, a contradiction. We have thus verified the claim.

We then choose a number $k=\max \left\{3, n_{l}\right\}$ of outer vertices as apices of an outer convex polygon $F_{\mathrm{o}}^{*}$ of $G^{\prime}$, as in the following two cases.

Case 1: $n_{l} \geq 3$.

In this case, as an apex, we choose an outer vertex $v$ of $G^{\prime}$ from each triconnected component $H_{j}$ corresponding to a leaf of $T$; $v$ must be other than the vertices of the separation pair with respect to $H_{j}$. We set these $n_{l}$ vertices chosen from all leaves of $T$ as the apices of $F_{\mathrm{o}}^{*}$. (In Fig. [8)(c), there are three triconnected components $H_{1}, H_{2}$ and $H_{3}$ corresponding to leaves of $T$ in Fig. 8 (d), and for example the three vertices depicted as white circles are chosen from $H_{1}, H_{2}$ and $H_{3}$.)

Case 2: $n_{l}=2$.

In this case, as apices, we first choose two outer vertices from the two leaves similarly as in Case 1 above. We then choose an arbitrary outer vertex other than the two vertices as an apex of $F_{\mathrm{o}}^{*}$. We set these three vertices as the apices of $F_{\mathrm{o}}^{*}$. (The triconnected component decomposition tree $T$ of $G^{\prime}$ for the graph $G$ in Fig. 1 has two leaves, and for example the three vertices depicted as white circles are chosen as outer apices.)

We finally claim that the $k$-gon $F_{\mathrm{o}}^{*}$ defined above is extendable. We shall show that Conditions (a)-(c) in Lemma 1 hold for $F_{\mathrm{o}}^{*}$. Since $G^{\prime}$ is internally triconnected, both Conditions (a) and (c) hold. It thus suffices to show that Condition (b) holds for $F_{\mathrm{o}}^{*}$. 
Suppose for a contradiction that Condition (b) does not hold for $F_{\mathrm{o}}^{*}$. Let $P_{1}$, $P_{2}, \cdots, P_{k}$ be the $k$ paths in $F_{\mathrm{o}}^{*}$, each corresponding to a side of the polygon $F_{\mathrm{o}}^{*}$. Since Condition (b) does not hold for $F_{\mathrm{o}}^{*}$, one of the following two cases occurs. Case (i): $G^{\prime}-F_{\mathrm{o}}\left(G^{\prime}\right)$ has a connected component $H$ such that all the outer vertices adjacent to vertices in $H$ lie on a single path $P_{i}$, as illustrated in Fig. 3(a).

Case (ii): two outer vertices in a path $P_{i}$ are joined by an inner edge, as illustrated in Fig. 3(b).

We first consider Case (i). Since $G^{\prime}$ is internally triconnected, $H$ is contained in a triconnected component $H_{j}$ of $G^{\prime}$ and $H_{j}$ must be a leaf of $T$. Then none of the outer vertices in $H_{j}$ other than the separation pair would be chosen as an outer apices, contrary to the definition of $F_{0}^{*}$.

We then consider Case (ii). Let $(u, v)$ be the innermost one among all the inner edges satisfying Case (ii), and let $H_{j}$ be the triconnected component containing edge $(u, v)$. Then $\{u, v\}$ is a separation pair of $G^{\prime}$ and $H_{j}$ is a leaf of $T$. None of the outer vertices in $H_{j}$ other than $u$ and $v$ is an apex of $F_{\mathrm{o}}^{*}$, contrary to the definition of $F_{\mathrm{o}}^{*}$.

Given a plane graph $G$ together with an extendable outer convex polygon $F_{\mathrm{o}}^{*}$, one can find in linear time a convex drawing of $G$ which is an extension of $F_{\mathrm{o}}^{*}$ [1,7]. Therefore, by Theorem 1 and Lemmas 2, 4and 5, we have the following theorem.

Theorem 2. Let $G$ be an internally triconnected graph, let $T$ be a triconnected component decomposition tree of a contracted graph $G^{\prime}$ of $G$, and let $n_{l}$ be the number of leaves of $T$. Then one can find a convex drawing of $G$ having the minimum number of outer apices in linear time, and the minimum number is equal to $\max \left\{3, n_{l}\right\}$.

\section{Conclusions}

In this paper, we newly formalize a necessary and sufficient condition for a plane graph $G$ to have a convex drawing, and show that a convex drawing having the minimum number of outer apices can be found in linear time.

In a convex grid drawing, all the vertices of $G$ are put on grid points. It is known that if either $G$ is triconnected or a triconnected component decomposition tree $T$ of a contracted graph $G^{\prime}$ of $G$ has at most three leaves then $G$ has a convex grid drawing of size $(n-1) \times(n-1)$ where $n$ is the number of vertices in $G[2,6$. The remaining problem is to obtain a good upper bound on the size of a convex grid drawing of $G$ for which $T$ has four or more leaves.

\section{References}

1. N. Chiba, T. Yamanouchi and T. Nishizeki, Linear algorithms for convex drawings of planar graphs, in Progress in Graph Theory, J. A. Bondy and U. S. R. Murty (Eds.), Academic Press, pp. 153-173 (1984).

2. M. Chrobak and G. Kant, Convex grid drawings of 3-connected planar graphs, International Journal of Computational Geometry and Applications, 7, pp. 211-223 (1997). 
3. H. de Fraysseix, J. Pach and R. Pollack, How to draw a planar graph on a grid, Combinatorica, 10, pp. 41-51 (1990).

4. G. Di Battista, P. Eades, R. Tamassia and I. G. Tollis, Graph Drawing, Prentice Hall, NJ (1999).

5. J. E. Hopcroft and R. E. Tarjan, Dividing a graph into triconnected components, SIAM J. Compt., 2, 3, pp. 135-138 (1973).

6. K. Miura, M. Azuma and T. Nishizeki, Canonical decomposition, realizer, Schnyder labeling and orderly spanning trees of plane graphs, International Journal of Fundations of Computer Science, 16, 1, pp. 117-141 (2005).

7. T. Nishizeki and Md. S. Rahman, Planar Graph Drawing, World Scientific, Singapore (2004).

8. W. Schnyder, Embedding planar graphs on the grid, Proc. 1st Annual ACM-SIAM Symp. on Discrete Algorithms, San Francisco, pp. 138-147 (1990).

9. C. Thomassen, Plane representations of graphs, in Progress in Graph Theory, J. A. Bondy and U. S. R. Murty (Eds.), Academic Press, pp. 43-69 (1984). 